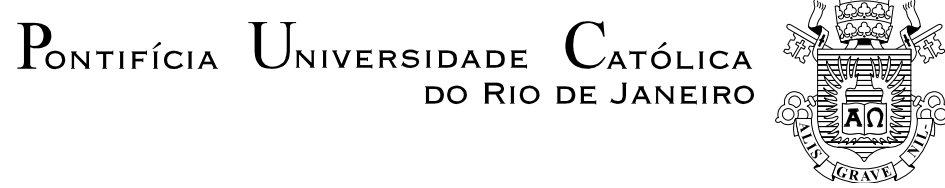

Neli Maria Castro de Almeida

Labirintos e mosaicos: institucionalização da infância com deficiência

TESE DE DOUTORADO

Tese apresentada ao Programa de Pós-Graduação em Serviço Social da PUC-Rio como requisito parcial para obtenção do título de Doutor em Serviço Social.

Orientadora: Profạ. Irene Rizzini

Rio de Janeiro

Agosto de 2012 


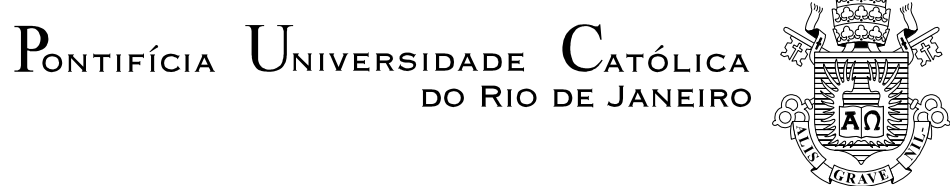

Neli Maria Castro de Almeida

\section{Labirintos e mosaicos: institucionalização da infância com deficiência}

Tese apresentada ao Programa de Pós-Graduação em Serviço Social da PUC-Rio como requisito parcial para obtenção do título de Doutor em Serviço Social. Aprovada pela Comissão Examinadora abaixo assinada.

Profa. Irene Rizzini

Orientadora

Departamento de Serviço Social - PUC - Rio

Profa. Sueli Bulhões da Silva

Departamento de Serviço Social - PUC - Rio

Profa. Denise Pini Rosalem da Fonseca Departamento de Serviço Social - PUC - Rio

Prof. Eduardo Mourão Vasconcelos Universidade Federal do Rio de Janeiro

Prof. Pedro Gabriel Godinho Delgado Universidade Federal do Rio de Janeiro

Profa Mônica Herz

Vice-Decana do Centro de Ciências Sociais - PUC - Rio

Rio de Janeiro, 02 de agosto de 2012 
Todos os direitos reservados. É proibida a reprodução total ou parcial do trabalho sem autorização da Universidade, da autora e do orientador.

\section{Neli Maria Castro de Almeida}

Psicóloga, formada pelo Instituto de Psicologia da Universidade Federal do Rio de Janeiro, Mestre em Psicologia Social e das Organizações pelo Instituto Superior de Ciências do Trabalho e da Empresa - ISCTE Lisboa, Portugal. Professora do Instituto Federal de Educação, Ciência e Tecnologia do Rio de Janeiro nos Cursos de Farmácia, Fisioterapia e Terapia Ocupacional. Coordenadora do Programa de Extensão do IFRJ/Campus Realengo. Atua nas áreas da Reforma Psiquiátrica brasileira, direitos humanos dos pacientes psiquiátricos e de pessoas com deficiência.

Ficha catalográfica

Almeida, Neli Maria Castro de

Labirintos e mosaicos: institucionalização da infância com deficiência / Neli Maria Castro de Almeida orientadora: Irene Rizzini. - 2012.

225 f. ; $30 \mathrm{~cm}$

Tese (doutorado)-Pontifícia Universidade Católica do Rio de Janeiro, Departamento de Serviço Social, 2012

Inclui bibliografia.

1. Serviço social - Teses. 2. Crianças e adolescentes. 3. Deficiência. 4. Deficiência mental. 5. Abrigamento. 6. Institucionalização. I. Rizzini, Irene. II. Pontifícia Universidade Católica do Rio de Janeiro. Departamento de Serviço Social. III. Título. 
A Adriana, Alessandra, Cosme, Anastácio, Renata, Filomena, Estelita, Alan, Ana Paula, Mirthes, Penha, Mônica (in memorian), Maria, Rogério, Marcelo, por tudo que me ensinaram com suas experiências de vida... 


\section{Agradecimentos}

Para a finalização desta tese muitas pessoas participaram de forma especial. Pude viver nesse período, momentos de expressa solidariedade de amigos, de profissionais que se envolveram com este projeto, e sem as quais, realmente, eu não teria conseguido chegar neste ponto do caminho.

A essas pessoas gostaria de me dirigir diretamente e expressar meu profundo, e para sempre, agradecimento.

Em especial, à profa. Ana Quiroga pelo acompanhamento feito nas etapas iniciais de elaboração da tese, permitindo o acesso a uma ampla literatura de suporte às ideias apresentadas nesta trabalho acadêmico.

À profa. Inez Teresinha Stampa, pelo seu trabalho atencioso na coordenação da Pós-Graduação em Serviço Social da PUC, que acolheu e acompanhou o percurso desta tese.

À Paula Caldeira que aceitou fazer a revisão em um tempo exíguo, já antecipando as horas das madrugadas, o trabalho intensivo e exaustivo. O seu trabalho rigoroso e delicado se presentificou neste percurso.

À Janaína Dória, o meu agradecimento sem palavras à altura por tamanha solidariedade e companheirismo neste momento final da tese.

À Mira Wengert pela franqueza da sua crítica à Figura 1, e pelo presente da nova Figura que ilustra uma das páginas desta tese.

Aos meus amigos queridos, que todo esse tempo de dedicação máxima às exigências da elaboração desta tese produziu um importante intervalo de encontros: a Erô, ao Geo, a Bernadete, meu expresso desejo de retorno ao acolhimento da amizade.

Agradeço o apoio incondicional de meus pais, Luiz e Guiomar, que têm sido fundamental nessa caminhada da vida. 
No âmbito profissional, agradeço ao Instituto Franco Basaglia, na representação dos seus Presidentes, Pedro Gabriel Delgado, Elaine Savi, Domingos Sávio e Lisete Vaz, pela possibilidade de ter vivido "a minha melhor oficina terapêutica".

Gostaria de agradecer ao Pedro Gabriel Godinho Delgado, à época, Coordenador do Programa Nacional de Saúde Mental do Ministério da Saúde, pela ação exitosa junto ao Conselho Nacional de Pesquisa - $\mathrm{CNPq}$ - que permitiu o lançamento de editais de apoio a linhas de pesquisas em áreas deficitárias do Sistema Único de Saúde. Esta ação, em específico, ensejou o apoio do CNPq à pesquisa "Do confinamento ao acolhimento: mudando a prática de institucionalização de crianças e adolescentes portadores de necessidades especiais do estado do Rio de Janeiro" desenvolvida pelo CIESPI/PUC-Rio, e coordenada pela profa. Irene Rizzini. A minha participação nesta pesquisa foi fundamental para a elaboração posterior da tese.

Imensamente agradeço a toda equipe da Pesquisa "Do confinamento ao acolhimento", Luciene, Michelle, Ana Gabriela, Verônica, Nathalia, Marcelo, Fábio e Aline, pela importante oportunidade de aprendizagem.

Assim, segue meu agradecimento ao Conselho Nacional de Pesquisa pelo apoio à pesquisa realizada junto ao CIESPI/PUC-Rio, e depois à finalização do Curso de Doutorado. Assim como agradecer à PUC-Rio pelos auxílios concedidos, sem os quais este trabalho não teria se realizado.

E, finalmente, três agradecimentos muito especiais: à Irene Rizzini pela orientação neste percurso e, sobretudo, por ter compreendido as minhas idiossincrasias no trabalho intelectual. À Irene, meu agradecimento para sempre.

Ao Octávio, por este acompanhamento de um tempo sempre presente.

As minhas queridas amigas Marta e Monique, em nome de uma amizade sem fim. 


\section{Resumo}

Almeida, Neli Maria Castro; Rizzini, Irene. Labirintos e mosaicos: institucionalização da infância com deficiência. Rio de Janeiro, 2012. 225p. Tese de Doutorado - Departamento de Serviço Social, Pontifícia Universidade Católica do Rio de Janeiro.

Esta tese tem por objetivo principal analisar os processos de produção da longa permanência de crianças e adolescentes com deficiência na rede assistencial de abrigamento. Partindo da descrição da própria experiência profissional, impulsionada pelas contribuições de Erving Goffman e Franco Basaglia, a autora introduz o conceito de hibridismo assistencial para analisar o problema da deficiência institucionalizada nas interfaces entre os campos da Psiquiatria e da Assistência Social. Tendo como campo de estudo a rede de abrigos específicos para a deficiência no estado do Rio de Janeiro, a autora utiliza metodologias quantitativas e qualitativas para analisar o quadro atual da assistência asilar para crianças e adolescentes com deficiência, definindo-se o seguinte corpus de análise: (1) elementos da historiografia da psiquiatria infantil brasileira, tendo por referência as contribuições de Michel Foucault. Nesta perspectiva, discute-se a figura histórica do Pavilhão-Escola Bourneville - dispositivo vinculado ao Hospício Nacional de Alienados e marco inaugural da psiquiatria infantil brasileira para a internação de crianças anormais; (2) dados do Datasus referentes às internações de crianças e adolescentes com deficiência, no período de 1998 a 2010, em território nacional, ressaltando-se a dimensão quantitativa do problema, e (3) entrevistas realizadas junto a agentes sociais do Sistema de Garantia dos Direitos da Criança e do Adolescente do estado do Rio de Janeiro, tendo por referência de análise o conceito de campo de Pierre Bourdieu, e de Complexo Tutelar, de Jacques Donzelot. O estudo conclui que (1) existe uma correlação entre os abrigos específicos e a história da institucionalização da deficiência mental, mantendo-se a figura híbrida do abrigo-hospital, (2) as atuais políticas de desinstitucionalização não vêm incluindo crianças e adolescentes com deficiência, sendo necessário rever o conceito de crônicos para crianças e adolescentes no regime de internação hospitalar e, (3) a presença da deficiência é um fator de maximização das práticas tutelares, o que é verificado a partir dos discursos dos agentes sociais. A autora articula o tema em uma agenda de interesse público e acadêmico, buscando contribuir para a superação do modelo assistencial centrado na longa permanência, na rede asilar, de crianças e adolescentes com deficiência.

\section{Palavras-chave}

Crianças e adolescentes; deficiência; deficiência mental; abrigamento; institucionalização 


\section{Abstract}

Almeida, Neli Maria Castro; Rizzini, Irene (Advisor). Labyrinths and mosaics: institutionalization of children with disabilities. Rio de Janeiro, 2012. 225p. DSc. Thesis - Departamento de Serviço Social, Pontifícia Universidade Católica do Rio de Janeiro.

This thesis aims at examining the processes that produces long-term placement of children and adolescents with mental disabilities in the shelter care facility network. The author begins hers analysis with the description of her own professional experience, driven by the contributions of Erving Goffman and Franco Basaglia. She introduces the concept of hibridismo assistencial (the idea of social assistance as an entangled reality) to analyze the problem of institutional disability looking at the interconnection between the areas of psychiatry and social work. Taking as her field of study the network of special purpose shelters (intended exclusively for children and youth with disabilities) in the State of Rio de Janeiro, the author combines quantitative and qualitative methodologies to analyze the current situation of the shelter care system for children and adolescents with disabilities, with the following corpus of analysis: (1) elements of the Brazilian historiography of child psychiatry, with reference to an analysis of the concept of power based on Michel Foucault. Under this perspective, the author discusses the historical figure of the Bourneville School - an institution linked to the National Asylum for the Insane and the founding landmark of the Brazilian child psychiatric for the hospitalization of the so called abnormal children, (2) Datasus data on hospitalization of children and adolescents with mental disabilities, highlighting the numerical dimensions of the problem, and (3) interviews with social agents connected to the System of Guarantee of the Rights of Children and Adolescents in the State of Rio de Janeiro, using as reference analysis the concepts of field by Pierre Bourdieu, and guardianship complex by Jacques Donzelot. Based on her study, the author concludes that: (1) there is a correlation between the existing shelters and the history of the institutionalization of children with mental disabilities as well as the presence of the 'hybrid' institution - the 'shelter-hospital', (2) the current policies aiming at closing down residential institutions have not included children and adolescents with disabilities, therefore it is necessary to critically review the concept of crônicos (chronically impaired) as referred to the young population that is hospitalized, and (3) based on testimonies of the social agents interviewed, it is possible to conclude that the presence of a disability becomes a factor of maximizing paternalistic practices. The author concludes her analysis linking some of the main issues to an agenda of academic and public interest, in an attempt to contribute to overcome old models centered on the long-term placement of children and adolescents with disabilities in institutions.

\section{Keywords}

Children and adolescents; disabilities; mental disabilities; sheltering; institutionalization. 


\section{Sumário}

1.Introdução

Parte I

O mundo vivido dos abrigos para a deficiência

2. Descrições e redescrições do problema: o fio condutor da experiência

2.1 "Pathos" e memórias de uma aprendiz: com quantos espantos se constrói um problema de estudo?

2.2 A Colônia Juliano Moreira dos anos 80, e o lugar da pessoa com deficiência mental

2.3 A Clínica Saint Roman: o primeiro contato com a infância psiquiatrizada

2.4 A Clínica Amendoeiras: o primeiro censo psicossocial da infância manicomializada

2.5 O Levantamento da rede de assistência para as pessoas com deficiência em situação de longa permanência no município do Rio de Janeiro

2.6 A deficiência mental e a Reforma Psiquiátrica brasileira

2.7 A experiência do projeto De Volta à Cidadania

2.8 A pesquisa com foco em crianças e adolescentes com deficiência em situação de abrigamento

3. De volta à cidadania: a intervenção e os desdobramentos da experiência

3.1 A intervenção: um breve histórico do projeto De Volta à Cidadania

3.2 Eixos estruturantes do projeto De Volta à Cidadania

3.3 A construção da crítica aos abrigos específicos para a deficiência

3.4 Desdobramentos da experiência: "fazer casa", novos conceitos, novas metodologias de cuidado

Parte II

Labirintos e mosaicos da longa permanência: o hibridismo assistencial e os discursos da proteção social

4. A infância com deficiência e o hibridismo assistencial

4.1 Uma aproximação ao conceito de deficiência e suas complexidades

4.2 A deficiência e a psiquiatria: o encontro da deficiência com o pensamento científico

5. A rede assistencial e a longa permanência da deficiência

5.1 Os dados numéricos da internação de crianças e adolescentes com deficiência na rede asilar 
5.2 O custo financeiro das internações de crianças e adolescentes na rede asilar

5.3 Crianças e adolescentes institucionalizados: raça, gênero e regionalização

6. A longa permanência e as práticas discursivas da proteção social à infância com deficiência

6.1 A interpretação dos dados qualitativos: episteme e questões metodológicas

6.20 inventário temático da longa permanência da deficiência institucionalizada

6.3 Primeira síntese temática - O poder e o complexo tutelar

6.4 Segunda síntese temática - O mal-estar da consciência crítica e o nada a fazer

158

6.5 Terceira síntese temática - A família no espelho: o descuido como peça de acusação

168

6.6 Quarta síntese temática - Cuidado, gênero e feminização da família

176

6.7 Quinta síntese temática - Em nome do interesse maior da criança

6.8 Sexta síntese temática - A infância com deficiência e a sua longa permanência no abrigo

6.9 Sétima síntese temática - O abrigo como máscara do problema. Onde está o começo de tudo?

188

6.10 Oitava síntese temática - A questão social como um enigma no interior do Sistema de Garantia de Direito

7. Conclusão

O caminho feito e os novos problemas de pesquisa

8. Referências Bibliográficas 202

Anexos 211 


\section{Lista de Figuras}

Figura 1- Fluxo da Regulação da Internação Hospitalar/AlH da morbidade Doenças do Sistema Nervoso-Capítulo VI/CID10- com incidência do SUAS, 2012.

Figura 2- № AlH pagas por morbidade: Transtornos mentais e comportamentais Capítulo V/CID 10, na faixa etária menor de 1 ano a 19 anos, $1998 / 2001 / 2006 / 2010$

Figura 3- Evolução do № AlH de Longa Permanência, por morbidade - Transtornos mentais e comportamentais - Capítulo V/CID 10, na faixa etária menor de 1 ano a 19 anos, 1998/2010

Figura 4- Evolução do № AlH Pagas por morbidade: Doenças do Sistema Nervoso - Capítulo VI/CID10, na faixa etária menor de 1 ano a 19 anos, 1998, 2001, 2006 e 2010

Figura 5- Evolução do № AlH de longa permanência por morbidade: Doenças do Sistema Nervoso - Capítulo VI/CID 10, na faixa etária menor de 1 ano a 19 anos, 1998 e 2010

Figura 6- Evolução do № AlH paga por morbidade: Transtornos mentais e comportamentais e Doenças do Sistema Nervoso Capítulos V e VI/CID 10, na faixa etária menor de 1 ano a 19 anos, 1998 e 2010

Figura 7- Evolução do № AlH de longa permanência por morbidade: Transtornos mentais e comportamentais e Doenças do Sistema Nervoso Capítulos V e VI / CID 10, na faixa etária menor de 1 ano a 19 anos, 1998 e 2010

Figura 8- Valor total $(\mathrm{R} \$)$ de $\mathrm{AlH}$ pagas, por morbidade: Transtornos mentais e comportamentais e Doenças do Sistema Nervoso Capítulos V e VI/CID 10, nas faixas etárias menor de 1 ano a 19 anos, 2010

Figura 9- Valor total $(\mathrm{R} \$)$ de AlH pagas, por morbidade: Transtornos mentais e comportamentais - Capítulo V /CID 10, nas faixas etárias menor de 1 ano, 1 a 4 anos, 5 a 9 anos, 15 a 19 anos, 1998 e 2010

Figura 10- Valor total $(\mathrm{R} \$)$ de AlH pagas, por morbidade: Doenças do Sistema Nervoso - Capítulo VI /CID 10, nas faixas etárias menor de 1 ano, 1 a 4 anos, 5 a 9 anos, 15 a 19 anos, 1998 e 2010 
Figura 11- Valor total $(\mathrm{R} \$)$ de $\mathrm{AlH}$ pagas por morbidade: Transtornos mentais e comportamentais e Doenças do Sistema Nervoso - Capítulos V e VI/CID 10, nas faixas etárias menor de 1 ano, 1 a 4 anos, 5 a 9 anos, 15 a 19 anos, 1998 e 2010

Figura 12- Valor total $(\mathrm{R} \$)$ de AlH pagas, por morbidade: Capítulo V/CID10 Transtornos mentais e comportamentais com inclusão e exclusão das AlH por uso de substâncias psicoativas, nas faixas etárias menor de 1 ano, 1 a 4 anos, 5 a 9 anos, 15 a 19 anos, 1998

Figura 13- Variação percentual do valor $(R \$)$ total das $A l H$ pagas por morbidade: Transtornos mentais e comportamentais e Doenças do Sistema Nervoso Capítulo V e VI/CID10, 1998 e 2010.

Figura 14- Valor total $(\mathrm{R} \$)$ de $\mathrm{AlH}$ pagas por morbidade: Transtornos mentais e comportamentais e Doenças do Sistema Nervoso, Capítulo V e VI/CID10, na faixa etária menor de 1 ano a 19 anos, 1998 e 2010

Figura 15- Número total de AlH pagas, por morbidade: Transtornos mentais e comportamentais - Capítulo V/CID10, por cor/raça, nas faixas etárias menor de 1 ano, 1 a 4 anos, 5 a 9 anos, 15 a 19 anos, 2010

Figura 16- Número total de AlH pagas, por morbidade: Doenças do Sistema Nervoso - Capítulo VI/CID10, por cor/raça, nas faixas etárias menor de 1 ano, 1 a 4 anos, 5 a 9 anos, 15 a 19 anos, 2010

Figura 17- Número de AlH/Internação por morbidade: Transtornos mentais e comportamentais e Doenças do Sistema Nervoso Capítulos V e VI/CID10, por sexo, na faixa etária menor de 1 a 19 anos, 1998 e 2010

Figura 18- Valor $(\mathrm{R} \$)$ total de AlH Pagas por morbidade: Transtornos mentais e comportamentais e Doenças do Sistema Nervoso - Capítulos $\mathrm{V}$ e VI/CID 10, por região, na faixa etária de menor de 1 ano a 19 anos, 1998

Figura 19- Valor $(\mathrm{R} \$)$ total de AlH Pagas por morbidade: Transtornos mentais e comportamentais e Doenças do Sistema Nervoso- Capítulos V e VI/CID 10, por região, na faixa etária de menor de 1 ano a 19 anos, 2010

Figura 20- Valor $(\mathrm{R} \$)$ total de AlH Pagas por morbidade: Transtornos mentais e comportamentais - Capítulo V/CID10, por região, na faixa etária menor de 1 ano a 19 anos, 1998 e 2010 
Figura 21- Valor $(\mathrm{R} \$)$ total de AlH Pagas por morbidade: Doenças do Sistema Nervoso- Capítulo VI/CID10, por região, na faixa etária menor de 1 ano a 19 anos, 1998 e 2010

Figura 22- Número de AlH pagas, na especialidade Psiquiatria, por natureza jurídica, segundo regiões, 1998

Figura 23- Número de AlH pagas, na especialidade Psiquiatria, por natureza jurídica, segundo regiões, 2010

Figura 24- Número de AlH pagas, na especialidade de Cuidados Prolongados (crônicos), por natureza jurídica, segundo regiões, 1998

Figura 25- Número de AlH pagas, na especialidade Cuidados Prolongados (crônicos), por natureza jurídica, segundo regiões, 2010

Figura 26- Número de AlH pagas, na especialidade Cuidados Prolongados (crônicos), por natureza jurídica, Região Sudeste, 1998

Figura 27- Número de AlH pagas, na especialidade Cuidados Prolongados (crônicos), por natureza jurídica, Região Sudeste, 2010 


\section{Lista de Tabelas}

Tabela 1: AlH Paga e AlH Tipo 5, por morbidade: Transtornos mentais e comportamentais - Capítulo V/CID10, na faixa etária menor de 1 a 19 anos, 1998 e 2010

Tabela 2: AlH Paga e AlH Tipo 5, por morbidade: Doenças do Sistema Nervoso - Capítulo VI / CID 10, na faixa etária menor de 1 ano a 19 anos, 1998 e 2010.

Tabela 3: Valor $(\mathrm{R} \$)$ de AlH pagas por morbidade: Capítulo V/CID 10 - Transtornos mentais/comportamentais, nas faixas etárias menor de 1 ano, 1 a 4 anos, 5 a 9 anos, 10 a 14 anos, 15 a 19 anos, 1998.

Tabela 4: Valor (R\$) de AlH pagas por morbidade: Capítulo VI/CID 10 - Doenças do Sistema Nervoso, nas faixas etárias menor de 1 ano, 1 a 4 anos, 5 a 9 anos, 10 a 14 anos, 15 a 19 anos, 1998.

Tabela 5: Valor (R\$) de AlH pagas por morbidade: Capítulo V/CID 10 - Transtornos mentais/comportamentais, nas faixas etárias menor de 1 ano, 1 a 4 anos, 5 a 9 anos, 10 a 14 anos, 15 a 19 anos, 2010.

Tabela 6: Valor (R\$) de AlH pagas por morbidade: Capítulo VI/CID 10 - Doenças do Sistema Nervoso, nas faixas etárias menor de 1 ano, 1 a 4 anos, 5 a 9 anos, 10 a 14 anos, 15 a 19 anos, 2010.

Tabela 7: Valor médio $(\mathrm{R} \$)$ das AlH pagas por morbidade: Capítulo V/CID 10 - Transtornos mentais/comportamentais e Capítulo VI/CID 10 - Doenças do Sistema Nervoso, nas faixas etárias menor de 1 ano, 1 a 4 anos, 5 a 9 anos, 10 a 14 anos, 15 a 19 anos, 2010.

Tabela 8: Valor $(\mathrm{R} \$)$ total e percentual de diferenciação das $\mathrm{AlH}$ pagas nas morbidades Transtornos mentais e comportamentais e Doenças do Sistema Nervoso, Capítulo V e VI / CID 10, por faixa etária, nos anos de 1998 e 2010.

Tabela 9: Número AlH Pagas por morbidade: Transtornos mentais e comportamentais e Doenças do Sistema Nervoso, Capítulos V e

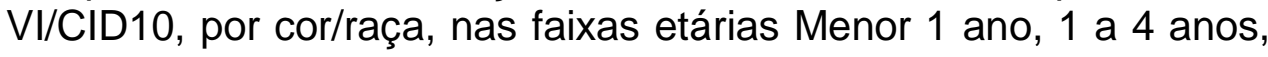
5 a 9 anos, 10 a 14 anos, 15 a 19 anos, 2010 
Tabela 10: Comparação entre o número e percentual de $\mathrm{AlH} /$ Internação, por morbidade: Transtornos mentais e comportamentais e Doenças do Sistema Nervoso, Capítulos V e VI/CID 10, por sexo, na faixa etária menor de 1 ano a 19 anos, 1998 e 2010

Tabela 11: Valor $(\mathrm{R} \$)$ total de $\mathrm{AlH}$ Pagas por morbidade: Transtornos mentais e comportamentais e Doenças do Sistema Nervoso- Capítulos $\mathrm{V}$ e VI/CID 10, por região, na faixa etária de menor de 1 ano a 19 anos, 1998 e 2010

Tabela 12: Número e percentual de AlH pagas, por natureza jurídica e por região, na modalidade Psiquiatria, 1998 e 2010.

Tabela 13: Número e percentual de AlH pagas, por natureza jurídica e por região, em Cuidados Prolongados (crônicos), 1998 e 2010.

Tabela 14: Número de AlH pagas, por natureza jurídica, em Cuidados Prolongados (crônicos), Região Sudeste, 1998.

Tabela 15: Número de AlH pagas, por natureza jurídica, em Cuidados Prolongados (crônicos), Região Sudeste, 2010. 


\section{Abreviaturas e Siglas}

AlH - Autorização de Internação Hospitalar

SIH - Sistema de Informações Hospitalares

SUS - Sistema Único de Saúde

SUAS - Sistema Único de Assistência Social

DATASUS - Banco de dados de informação em Saúde

CAPS - Centro de Atenção Psicossocial

ECA- Estatuto da Criança e do Adolescente

BIREME - Biblioteca Regional de Medicina

LILACS - Literatura Latino Americana e do Caribe em Ciências da Saúde 


\section{Anexos}

Anexo I - Parecer da Comissão de Ética em Pesquisa PUC-Rio referente à Pesquisa "Do confinamento ao acolhimento: mudando a prática de institucionalização de crianças e adolescentes portadores de necessidades especiais do estado do Rio de Janeiro".

Anexo II - Roteiro das entrevistas realizadas junto aos agentes do Sistema de Garantia dos Direitos de Crianças e Adolescentes do estado do Rio de Janeiro.

Anexo III - Dados de contextualização da Pesquisa "Do confinamento ao acolhimento: mudando a prática de institucionalização de crianças e adolescentes portadores de necessidades especiais do estado do Rio de Janeiro".

Anexo IV - Temas das publicações científicas sobre crianças e adolescentes com deficiência, base Lilacs-Brasil e América Latina - Ano 2012.

Anexo V- Distribuição dos temas sobre crianças e adolescentes com deficiência por Periódicos Científicos, segundo estado/país de origem, na base Lilacs - Brasil e América Latina, 2012.

Anexo VI - Tabelas 1 e 2 referentes à frequência das internações por especialidades Cuidados Prolongados e Psiquiatria em Unidades Assistenciais nos Estados de São Paulo e Rio Janeiro, nas faixas etárias menor de 1 ano a 19 anos, ano de 2006. 
Jogos de amarelinha

Jogos de amarelinha Que tempos tão distantes de uma infância sabida

Fazer o grande desenho no chão,

As casas

Os quadrados,

Os retângulos, Sair da primeira casa, chegar à última casa

Chegar ao céu

Voltar tudo de novo O céu não era lugar para se ficar Era lugar de chegar para poder voltar

Jogos de amarelinha Lançar a pedra na casa certa

Pular de um pé Pular de dois pés Jogar a pedra para trás Acertar a casa sem olhar Fazer um caminho de ida e volta

E muitas vezes pegar a pedra

Lançar a pedra Jogos de amarelinha Quanta infância sabida! Pode uma criança viver sem o seu jogo de amarelinha?

Neli de Almeida 